\title{
Effects of branching position of alkyl side chains on ordering structure and charge transport property in thienothiophenedione- and quinacridone-based semiconducting polymers
}

\author{
Kohsuke Kawabata, Masahiko Saito, Noriko Takemura, Itaru Osaka and Kazuo Takimiya
}

To investigate the effect of the branching position of the alkyl groups on the side chain of semiconducting polymers, we synthesized two series of semiconducting polymers based on thienothiophene-2,5-dione (PTTD4Ts) and quinacridone (PQA2Ts). 2-Decyltetradecyl, 3-decylpentadecyl, 4-decylhexadecyl, and 5-decylheptadecyl groups were used, and the branching position was systematically varied from the second carbon from the backbone to the fifth carbon. These branched side chains are introduced into the thiophene ring for PTTD4Ts and the quinacridone unit for PQA2Ts. The polymer thin films exhibited small but clear differences in their optical absorption spectra, suggesting that the intermolecular interaction in the solid state varied based on the branching position. The grazing incident X-ray diffraction study revealed that the $\pi-\pi$ stacking $d$-spacing of both polymers decreased when the branching position was moved away from the backbones, indicating that the intermolecular interaction was enhanced. Therefore, regardless of the core where the alkyl groups were introduced, the branching position effectively improved the ordering structure of the polymers, which was most likely due to suppressed steric hindrance. Although PTTD4Ts did not exhibit a clear correlation between the branching position and charge carrier mobility, the mobility of PQA2Ts gradually increased as the branching position moved away from the backbone.

Polymer Journal (2017) 49, 169-176; doi:10.1038/pj.2016.103; published online 19 October 2016

\section{INTRODUCTION}

Solution-processable semiconducting polymers have been extensively studied as low-cost, flexible, and lightweight electronic materials for potential applications, including organic field-effect transistors (OFETs), ${ }^{1}$ organic light-emitting diodes, ${ }^{2}$ and organic photovoltaics. ${ }^{3}$ In the development of semiconducting polymers, the design of the conjugated backbone is of primary importance because it substantially affects the molecular orbital energies, ${ }^{4-6}$ bandgaps, ${ }^{7,8}$ and ordering structure $^{9,10}$ that determine the device performance of the polymers. To date, enormous effort has been focused on the exploration of backbone structures (that is, new conjugated building units and their combinations). In parallel, flexible side chains are also important components of semiconducting polymers, and these side chains have a role in determining the ordering structures, the electronic properties, and their solubility in organic solvents. Pioneering studies of the side chains have been performed by McCullough ${ }^{11,12}$ and Rieke, ${ }^{13}$ in which they successfully controlled the regiochemistry of the alkyl side chains in poly(3-alkylthiophene)s (P3ATs) during the polymerization of asymmetrical 3-alkylthiophene monomers to yield head-to-tail regioregular P3ATs (rrP3ATs). In fact, rrP3ATs exhibited highly ordered structures with lamellar and $\pi-\pi$ stacking structures in a thin film. However, regiorandom P3ATs (raP3ATs) exhibited an amorphous-like or disordered structure, which resulted in rrP3ATs possessing high electrical properties, such as conductivity and hole mobility. ${ }^{14}$ The placement, length, and topology of the side chain are also crucial factors for the ordering structure $e^{15-17}$ and the backbone orientation, such as edge-on and face-on, which affect the direction of charge transport. ${ }^{18}$

Recently, branched alkyl groups have been widely used as the side chain in semiconducting polymers because they provide better solubility than simple linear alkyl groups. In particular, for semiconducting polymers with donor-acceptor backbones that have strong intermolecular interactions, branched alkyl groups are necessary for solubility in organic solvents. ${ }^{19,20}$ Although such bulky branched alkyl groups efficiently increase the solubility, they suppress the aggregation or close interchain $\pi-\pi$ stacking of the polymers relative to the linear alkyl groups, resulting in inefficient charge transport. ${ }^{20}$ A recent study demonstrated that moving the branching position in the branched alkyl group away from the conjugated backbone reduced the steric effect and $\pi-\pi$ stacking distances, which enhanced the charge carrier mobilities in OFET devices. ${ }^{21-25}$ However, in most of these studies, branched alkyl groups are 
<smiles>[R]c1cc(C2=C3SC(=O)C(c4cc([R])c(-c5ccc(-c6ccc(C(C)(C)C)s6)s5)s4)=C3SC2=O)sc1C</smiles>

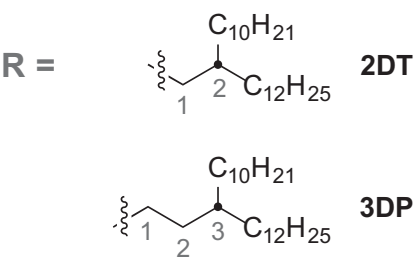<smiles>[R]n1c2ccc(-c3ccc(-c4ccc(C(C)(C)C)s4)s3)cc2c(=O)c2cc3c(cc21)c(=O)c1cc(C(C)(C)C)ccc1n3[R]</smiles>

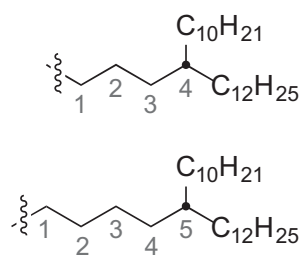

4DH

Figure 1 Chemical structures of PTTD4Ts, PQA2Ts, and branched alkyl side chains. A full color version of this figure is available at Polymer Journal online.

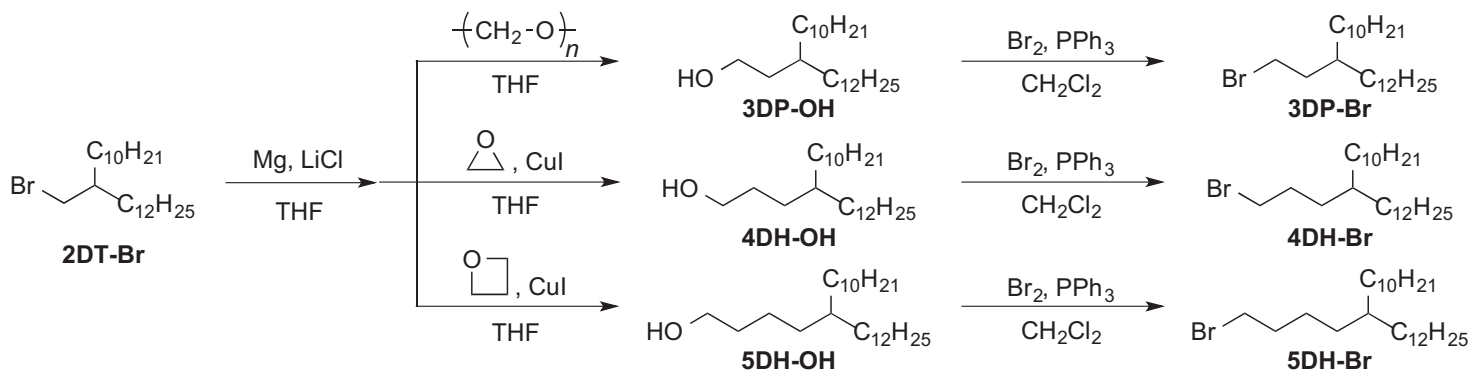

Scheme 1 Synthesis of branched alkyl groups. THF, tetrahydrofuran.<smiles>[R]c1cc(C2=C3SC(=O)C(c4cc([R])c(Br)s4)=C3SC2=O)sc1Br</smiles>

TTD2TBr2-R<smiles>[R]n1c2ccc(Br)cc2c(=O)c2cc3c(cc21)c(=O)c1cc(Br)ccc1n3[R]</smiles>

QABr2-R

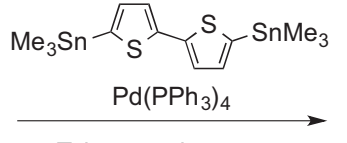

Toluene, microwave

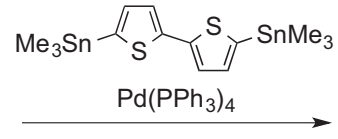

Toluene, microwave

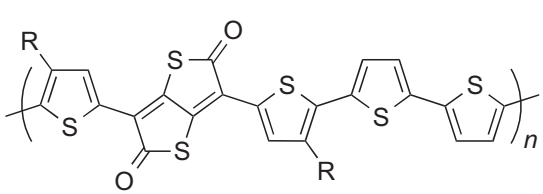

PTTD4T-R (R = 2DT, 3DP, 4DH, 5DH)

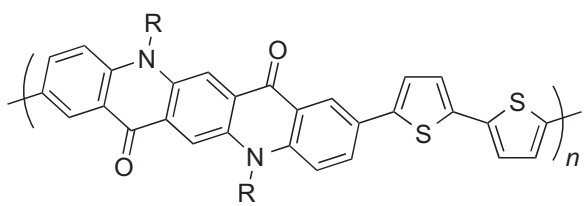

PQA2T-R (R = 2DT, 3DP, 4DH, 5DH)

Scheme 2 Synthesis of polymers.

introduced into the $\mathrm{N}$-position of limited cores, such as isoindigo and diketopyrrolopyrrole. ${ }^{26-28}$

Here, to further systematically study the branching position effect, we introduced a series of branched alkyl groups with different branching positions (that is, 2-decyltetradecyl (2DT), 3-decylpentadecyl (3DP), 4-decylhexadecyl (4DH), and 5-decylheptadecyl (5DH) groups; Figure 1) into the 3-position of the thiophene ring and the $N$-position of QA (quinacridone). Then, the 3-alkylthiophenes were combined with the TTD (thienothiophene2,5-dione) unit, which was copolymerized with the unsubstituted bithiophene unit (PTTD4Ts), and the alkylated QA units were also copolymerized with the unsubstituted bithiophene unit (PQA2Ts; Figure 1). The electronic properties, microstructure, and the OFET performance of the polymer thin films were characterized, and the correlation with the branching positions is discussed.

\section{RESULTS AND DISCUSSION}

The syntheses of the branched alkyl groups are shown in Scheme 1. The 2DT, 3DP, 4DH, and 5DH groups with branching positions at the second, third, fourth, and fifth carbon from the backbone were 
chosen for the side chains. In all of the cases, the branched parts are decyl and dodecyl groups. 3-Decylpentadecan-1-ol (3DP-OH), 4-decylhexadecan-1-ol (4DH-OH), and 5-decylheptadecan-1-ol (5DH-OH) were synthesized from 2-decyl-1-tetradecyl bromide (2DT-Br) via the formation of the Grignard reagent followed by treatment with paraformaldehyde, ${ }^{29}$ ethylene oxide, ${ }^{30}$ and oxetane, ${ }^{31}$ respectively. These alkyl alcohols were brominated to afford the corresponding bromides (that is, 3-decyl-1-pentadecyl bromide (3DP-Br), 4-decyl-1-hexadecyl bromide (4DH-Br), and 5-decyl-1heptadecyl bromide (5DH-Br)). These alkyl bromides were successfully introduced into the thiophene rings in the TTD-based monomers (TTD2TBr2s) and QA-based monomers (QABr2s) according to previously reported procedures. ${ }^{32,33}$ These monomers were copolymerized with 5,5'-bis(trimethylstannyl)-2,2'-bithiophene using the Migita-Kosugi-Stille coupling reaction in the presence of tetrakis(triphenylphosphine)palladium(0) to yield the corresponding polymers (PTTD4Ts and PQA2Ts), as shown in Scheme 2. The polymers were washed with methanol, hexane, and chloroform using a Soxhlet apparatus to remove the low molecular weight fraction. Then, the purified polymers were collected by chlorobenzene.

Table 1 Molecular weights of polymers ${ }^{a}$

\begin{tabular}{lcrr}
\hline Polymer & $\mathrm{M}_{n}(\mathrm{kDa})$ & $\mathrm{M}_{w}(\mathrm{kDa})$ & $\mathrm{M}_{w} / \mathrm{M}_{n}$ \\
\hline PTTD4T-2DT & 50 & 319 & 6.3 \\
PTTD4T-3DP & 47 & 567 & 12.1 \\
PTTD4T-4DH & 48 & 1883 & 39.2 \\
PTTD4T-5DH & 39 & 614 & 15.7 \\
& & & \\
PQA2T-2DT & 9 & 68 & 9.1 \\
PQA2T-3DP & 12 & 138 & 11.5 \\
PQA2T-4DH & 13 & 97 & 8.0 \\
PQA2T-5DH & 11 & 77 & 7.0 \\
\hline
\end{tabular}

Abbreviations: $\mathrm{DCB}$, o-dichlorobenzene; $M_{\mathrm{n}}$, number-average molecular weight; $M_{\mathrm{w}}$, weight-average molecular weight.

aDetermined by high-temperature gel permeation chromatography (GPC) using DCB as the eluent at $140{ }^{\circ} \mathrm{C}$ and calibrated using polystyrene standards.
By moving the branching point away from the backbone, the polymer solubility slightly decreased in both cases. The numberaverage molecular weights $\left(M_{\mathrm{n}}\right)$ of the polymers ranged from 39 to $50 \mathrm{kDa}$ for PTTD4Ts and 9 to $13 \mathrm{kDa}$ for PQA2Ts based on gel permeation chromatography using $o$-dichlorobenzene as the eluent at $140^{\circ} \mathrm{C}$ (Table 1). The small deviation of $M_{\mathrm{n}}$ in PTTD4Ts and PQA2Ts would reduce the effects of the molecular weight and should provide a fair comparison of the polymer properties. The large polydispersity indices of the polymers could be an artifact of the bimodal peaks in the gel permeation chromatography curves, which are most likely caused by strong aggregation of the polymers in solution (Supplementary Figure S1). ${ }^{32}$ On the basis of differential scanning calorimetry, all of the polymers were thermally stable because they did not exhibit a melting peak below $300^{\circ} \mathrm{C}$ (Supplementary Figure S2).

To investigate the electronic properties of the polymers, we first evaluated the molecular orbital energies via cyclic voltammetry using polymer thin films (Figure 2). In all the cases, reversible oxidation and

Table 2 Physicochemical properties of the polymer films

\begin{tabular}{lcccccc}
\hline Polymer & $\begin{array}{c}\mathrm{E}_{\text {red }} \\
(\mathrm{V})^{\mathrm{a}}\end{array}$ & $\begin{array}{c}\mathrm{E}_{\text {ox }} \\
(\mathrm{V})^{\mathrm{a}}\end{array}$ & $\begin{array}{c}\mathrm{E}_{\text {LUMO }} \\
(\mathrm{eV})^{\mathrm{b}}\end{array}$ & $\begin{array}{c}\mathrm{E}_{\text {HOMO }} \\
(\mathrm{eV})^{\mathrm{b}}\end{array}$ & $\begin{array}{c}\lambda_{\max } \\
(\mathrm{nm})\end{array}$ & $\begin{array}{c}\mathrm{E}_{g} \\
(\mathrm{eV})^{\mathrm{d}}\end{array}$ \\
\hline PTTD4T-2DT & -0.97 & 0.39 & -3.83 & -5.19 & 818 & 1.23 \\
PTTD4T-3DP & -0.98 & 0.40 & -3.82 & -5.20 & 858 & 1.22 \\
PTTD4T-4DH & -1.04 & 0.29 & -3.76 & -5.09 & 876 & 1.16 \\
PTTD4T-5DH & -1.01 & 0.32 & -3.79 & -5.12 & 871 & 1.20 \\
& & & & & & \\
PQA2T-2DT & -1.73 & 0.20 & -3.07 & -5.00 & 456 & 2.02 \\
PQA2T-3DP & -1.75 & 0.21 & -3.05 & -5.01 & 481 & 2.01 \\
PQA2T-4DH & -1.82 & 0.12 & -2.98 & -4.92 & 484 & 2.01 \\
PQA2T-5DH & -1.77 & 0.08 & -3.03 & -4.88 & 483 & 2.00 \\
\hline
\end{tabular}

Abbreviations: HOMO, highest occupied molecular orbital; LUMO, lowest unoccupied molecular orbital.

aReduction potential $\left(E_{\mathrm{red}}\right)$ and oxidation potential $\left(E_{\mathrm{ox}}\right)$ determined from the onset of the cyclic voltammogram.

bLUMO $\left(E_{L U M O}\right)$ and HOMO ( $\left.E_{\text {HOMO }}\right)$ energy levels estimated using the following equation: $E_{\text {LUMO }}=-4.80-e E_{\text {red }}, E_{\text {HOMO }}=-4.80-e E_{\text {ox }}$

$E$ LUMO $=-4.80-e E_{\text {red }}$
CAbsorption maximum.

dOptical bandgap estimated from the onset of the absorption spectrum.
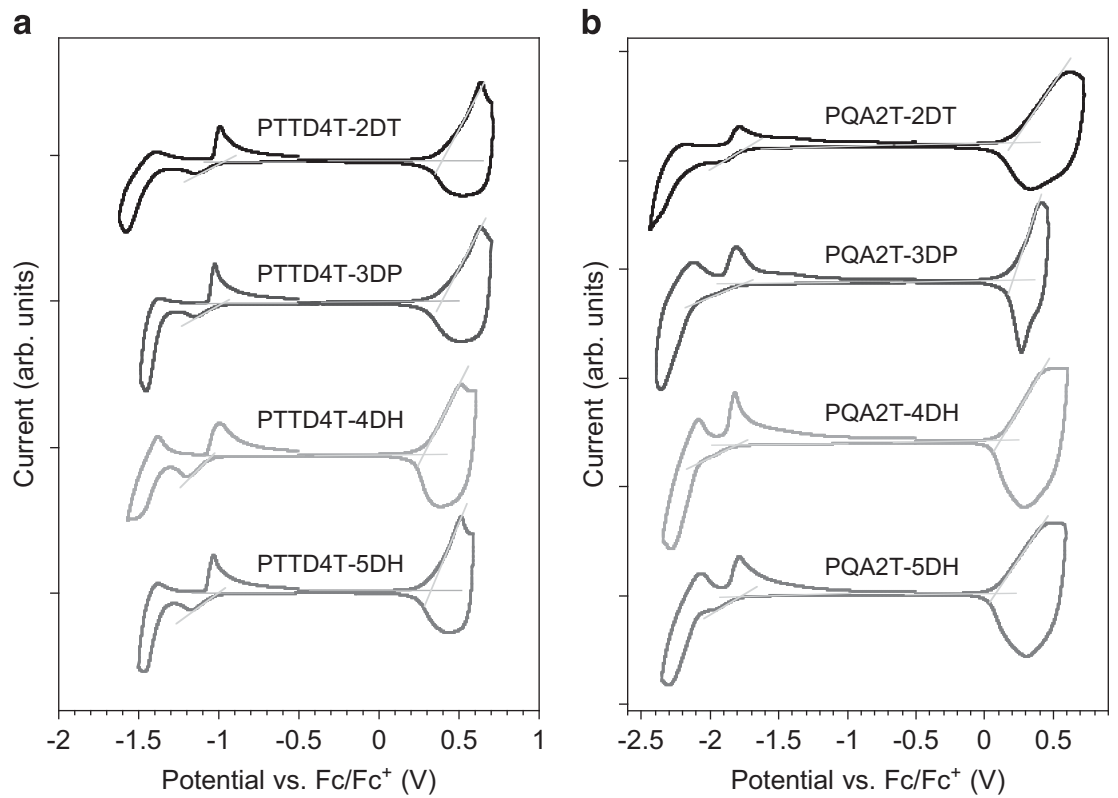

Figure 2 Cyclic voltammograms of the polymers. (a) PTTD4Ts, (b) PQA2Ts. A full color version of this figure is available at Polymer Journal online. 

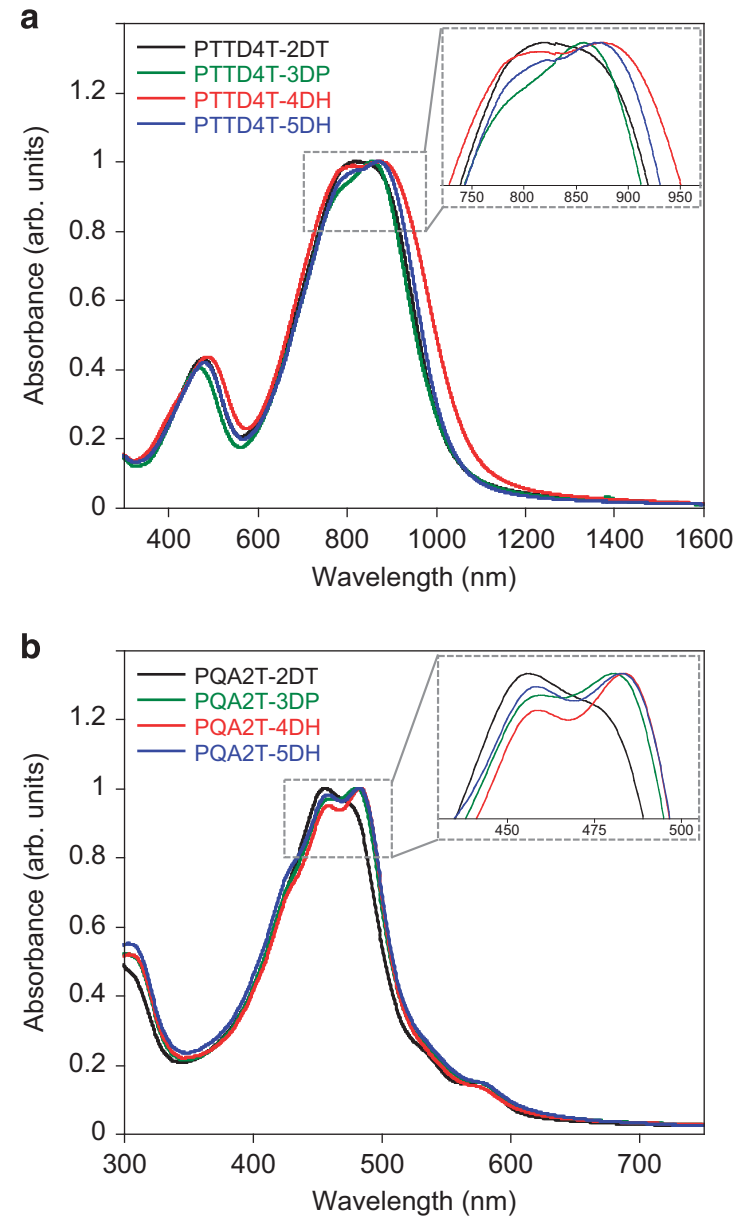

Figure 3 UV-Vis-NIR absorption spectra of the polymer thin films after annealing at $300{ }^{\circ} \mathrm{C}$. (a) PTTD4Ts and (b) PQA2Ts. Insets show the close-up of the peaks. UV-Vis-NIR, ultraviolet-visible-near infrared.

a

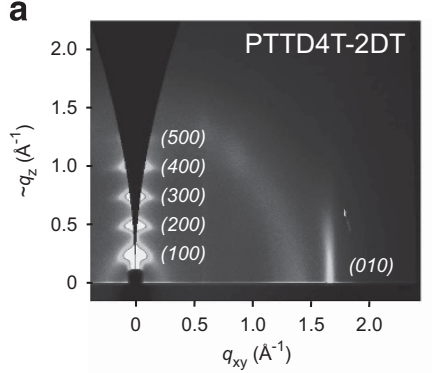

e

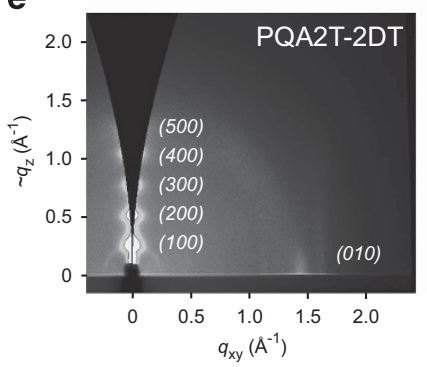

b

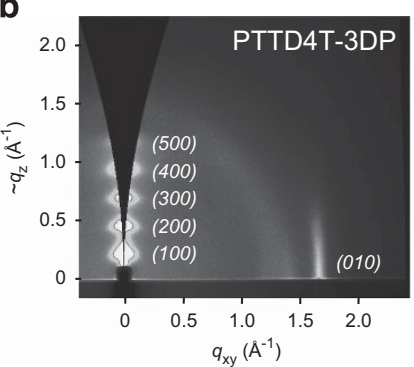

f

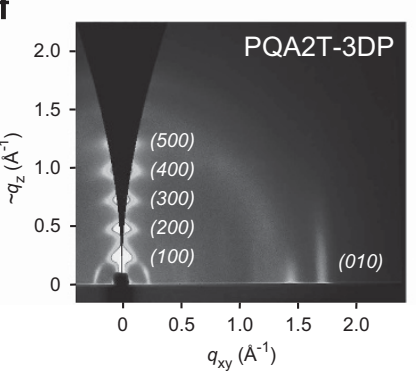

reduction signals were observed. The redox potentials and energy levels of the lowest unoccupied molecular orbital (LUMO) and the highest occupied molecular orbital (HOMO) are summarized in Table 2. Both the HOMO and LUMO energy levels $\left(E_{\mathrm{HOMO}}\right.$ and
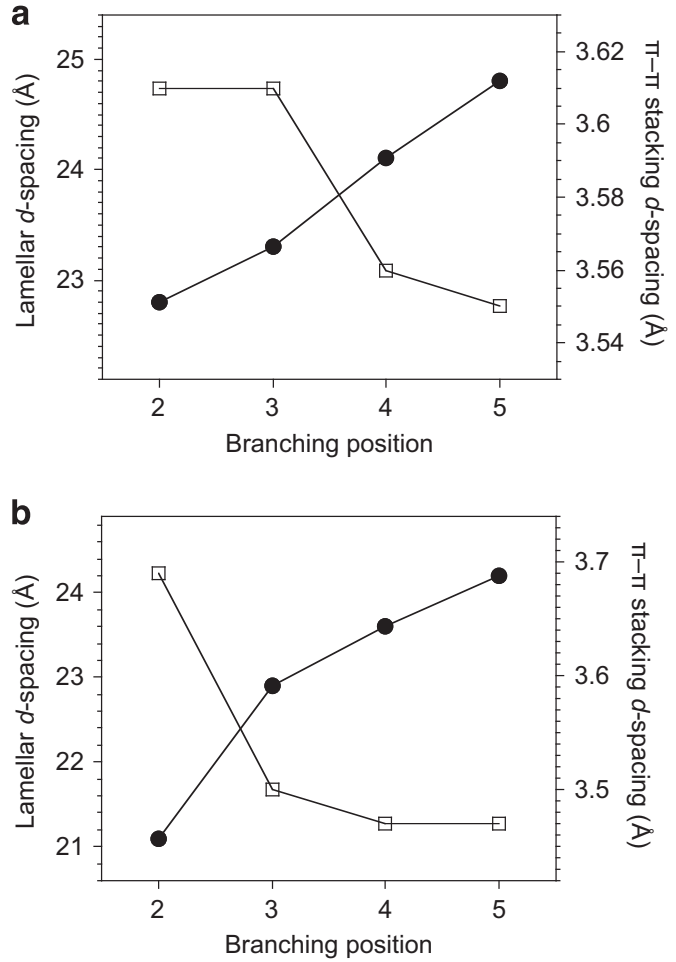

Figure 5 Lamellar (filled circle) and $\pi-\pi$ stacking (open square) $d$-spacings of the polymers calculated from the GIXD profiles along the $\sim q_{z}$ and $q_{x y}$ axes, respectively, as a function of the branching position (the number of carbon from the backbone). (a) PTTD4Ts and (b) PQA2Ts. GIXD, grazing incident X-ray diffraction.

C

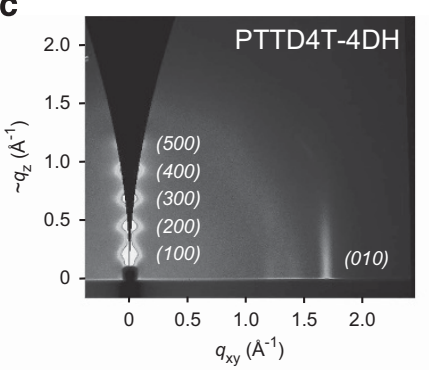

g

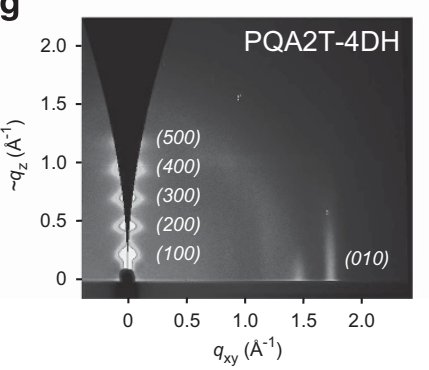

d

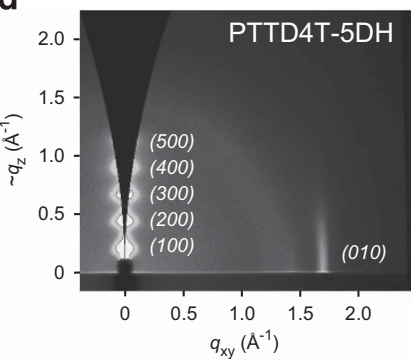

h

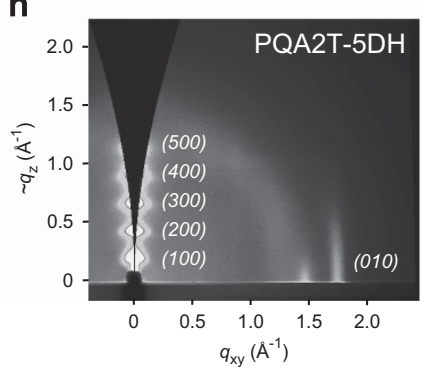

Figure 4 2D-GIXD patterns of the polymer thin films on ODTS-treated $\mathrm{SiO}_{2} / \mathrm{Si}$ substrates annealed at $300{ }^{\circ} \mathrm{C}$. (a) PTTD4T-2DT, (b) PTTD4T-3DP,

(c) PTTD4T-4DH, (d) PTTD4T-5DH, (e) PQA2T-2DT, (f) PQA2T-3DP, (g) PQA2T-4DH, and (h) PQA2T-5DH. 2D, two-dimensional; GIXD, grazing incident X-ray diffraction; ODTS, octadecyltriethoxysilane. A full color version of this figure is available at Polymer Journal online. 

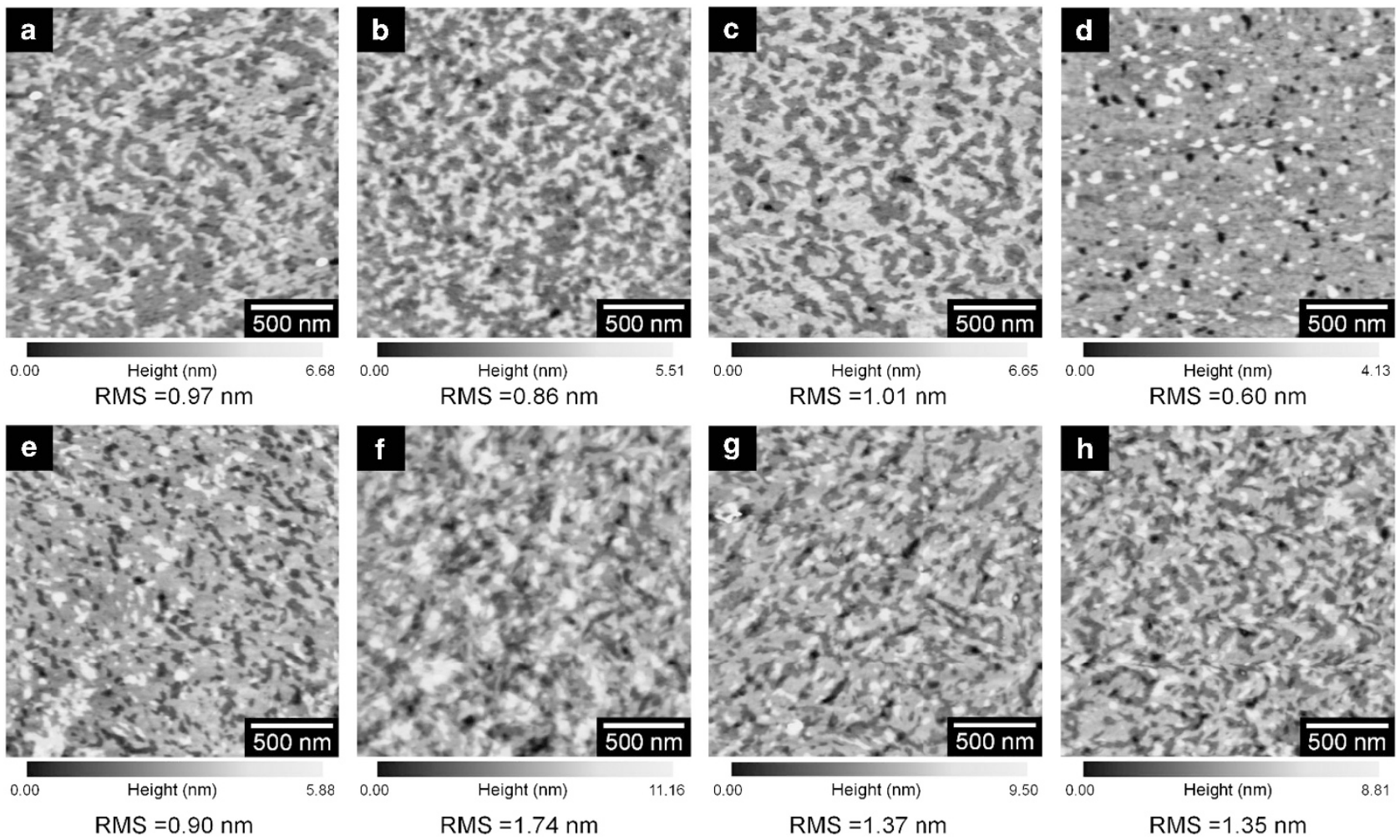

Figure 6 AFM height images and root-mean-square (RMS) roughness of the polymer thin films. (a) PTTD4T-2DT, (b) PTTD4T-3DP, (c) PTTD4T-4DH, (d) PTTD4T-5DH, (e) PQA2T-2DT, (f) PQA2T-3DP, (g) PQA2T-4DH, and (h) PQA2T-5DH. AFM, atomic force microscopy. A full color version of this figure is available at Polymer Journal online.

$E_{\mathrm{LUMO}}$ s) shifted upward from $3 \mathrm{DP}$ to $4 \mathrm{DH}$ when the branching position moved away from the backbone. The shift in the $E_{\text {HOMO }}$ was significant relative to that in the $E_{\mathrm{LUMO}}$. The $E_{\mathrm{HOMO}}$ of PTTD4T-2DT and -3DP was approximately $-5.2 \mathrm{eV}$, and the $E_{\mathrm{HOMO}}$ of PTTD4T-4DH and -5DH was approximately $-5.1 \mathrm{eV}$. Similarly, the $E_{\mathrm{HOMO}}$ of PQA2T-2DT and -3DP was approximately $-5.0 \mathrm{eV}$, and the $E_{\mathrm{HOMO}}$ of PQA2T-4DH and -5DH was approximately $-4.9 \mathrm{eV}$. These $E_{\mathrm{HOMO}}$ were consistent with those evaluated by photoemission yield spectroscopy (Supplementary Figure S3).

Next, we measured the UV-Vis-NIR (ultraviolet-visible-near infrared) absorption spectra of the polymer thin films (Figure 3) and the polymer solution (Supplementary Figure S4). The spectra of the thin films and solutions were similar, indicating that the polymers aggregated even in solution. The absorption maximum wavelengths and optical bandgaps extracted from the film absorptions are summarized in Table 2 . In both the cases, the polymers with different side chains exhibited similar absorption spectra with strong absorption bands at approximately $600-1000 \mathrm{~nm}$ for PTTD4Ts and 400-500 nm for PQA2Ts, and, therefore, similar optical bandgaps $\left(E_{\mathrm{g}} s\right)$ of approximately $1.2 \mathrm{eV}$ and $2.0 \mathrm{eV}$, respectively, were obtained. However, the peak tops shifted slightly. In PTTD4Ts, PTTD4T-3DP, -4DH, and -5DH exhibited clearer vibronic structures than PTTD4T-2DT. By moving the branching position away from the backbone, the absorption maximum $\left(\lambda_{\max }\right)$ of $818 \mathrm{~nm}$ for PTTD4T-2DT was red-shifted to $858 \mathrm{~nm}$ for PTTD4T-3DP, $876 \mathrm{~nm}$ for PTTD4T-4DH, and $871 \mathrm{~nm}$ for PTTD4T-5DH. This shift implies that PTTD4T-3DP, $-4 \mathrm{DH}$, and $-5 \mathrm{DH}$ possess better intermolecular interaction or ordering in the solid state than PTTD4T-2DT. A similar trend for the spectral difference as a function of the branching position was also observed for PQA2Ts. PQA2T-3DP, $-4 \mathrm{DH}$, and $-5 \mathrm{DH}$ exhibited clearer vibronic structures than PQA2T-2DT, and the $\lambda_{\max }$ of $456 \mathrm{~nm}$ for PQA2T-2DT was red-shifted to $481 \mathrm{~nm}$ for PQA2T-3DP, $484 \mathrm{~nm}$ for PQA2T-4DH, and $483 \mathrm{~nm}$ for PQA2T-5DH. Although the change in the $E_{\mathrm{g}}$ may not directly correlate with a change in the energy levels, the change in the vibronic structure may qualitatively be in agreement.

The microstructures of the polymer thin films were investigated using grazing incident X-ray diffraction (GIXD) measurements. Figure 4 shows the two-dimensional GIXD patterns of the polymer thin films annealed at $300^{\circ} \mathrm{C}$, and these films were prepared on octadecyltriethoxysilane-treated $\mathrm{Si} / \mathrm{SiO}_{2}$ substrates in the same manner as the fabrication of the OFET devices. In the two-dimensional GIXD patterns, both the PTTD4Ts and PQA2Ts thin films provided strong spot-like diffractions up to the fifth-order along the quasi- $q_{\mathrm{z}}$ axis, which correspond to a lamellar structure (h00), and slit-like diffractions along the $q_{\mathrm{xy}}$ axis, which correspond to a $\pi-\pi$ stacking structure (010). This result indicates that all of the polymers are crystalline. In addition, the polymer backbones are oriented in an edge-on manner on the substrate, which is regarded as a favorable thin film structure for lateral charge transport.

The lamellar and $\pi-\pi$ stacking $d$-spacings were extracted from the out-of-plane and in-plane profiles of the two-dimensional GIXD images (Supplementary Figure S5, Supplementary Table S1) and are plotted as a function of the branching position from the backbone, as shown in Figure 5. In both cases, the lamellar $d$-spacing increased monotonically as the branching position increased. In PTTD4Ts, the $\pi-\pi$ stacking $d$-spacing was $3.61 \AA$ for PTTD4T-2DT and -3DP, which decreased to $3.56 \AA$ for $-4 \mathrm{DH}$ and $3.55 \AA$ for $-5 \mathrm{DH}$. In PQA2Ts, the $\pi-\pi$ stacking $d$-spacings was $3.69 \AA$ for PQA2T-2DT, which decreased to $3.50 \AA$ for $-3 \mathrm{DP}$ and further decreased to $3.47 \AA$ for $-4 \mathrm{DH}$ and 
a

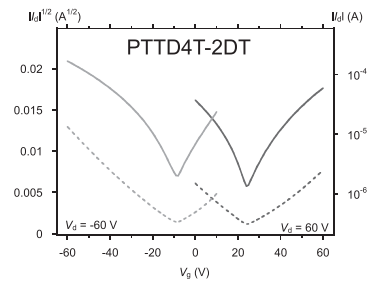

e

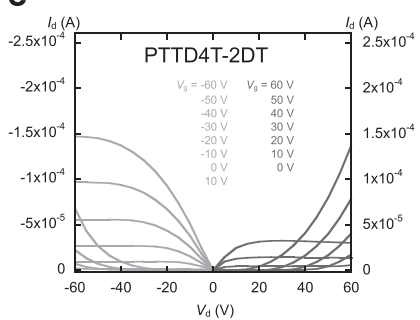

i

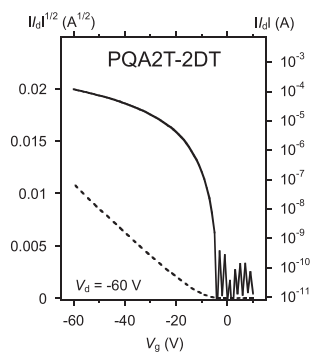

m

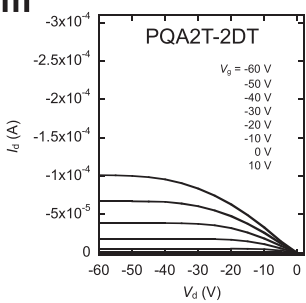

b

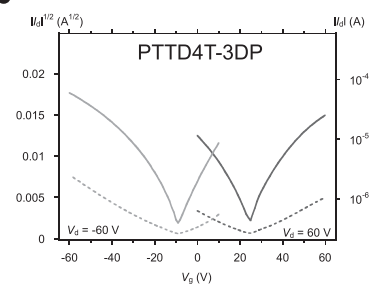

f

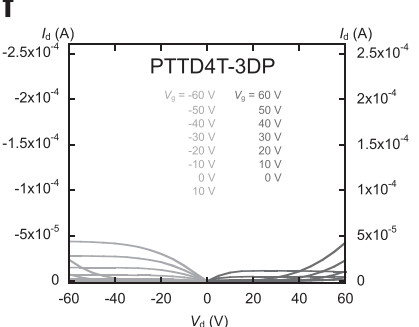

j

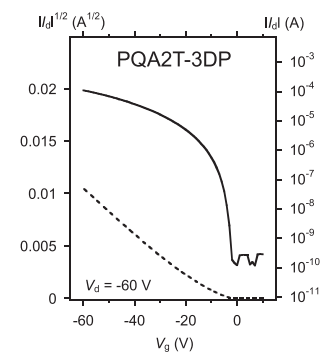

n

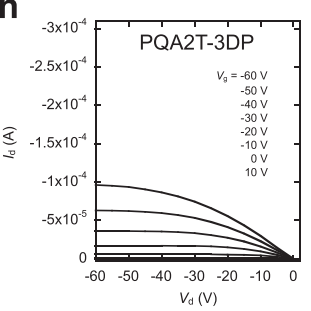

C

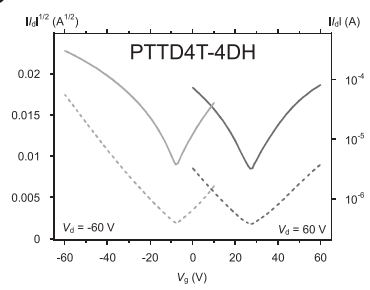

g

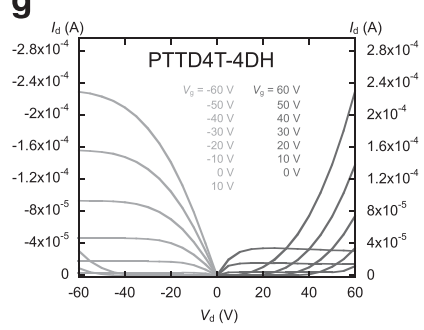

k

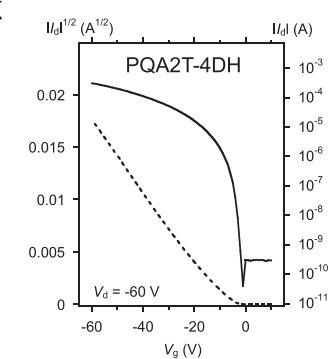

0

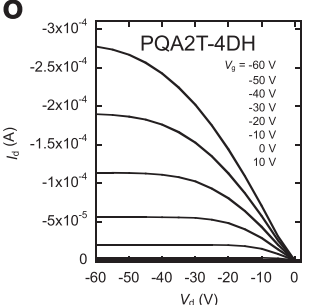

d

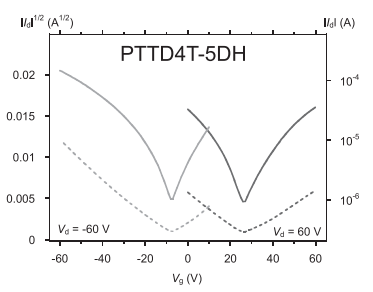

h

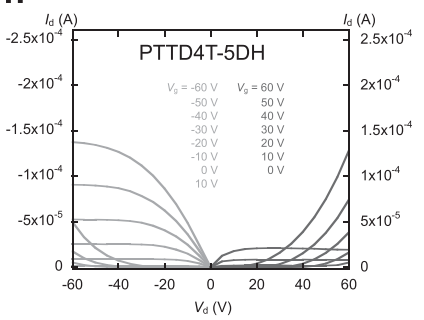

I

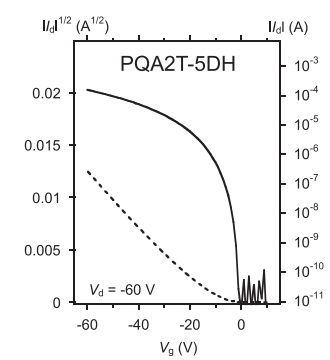

p

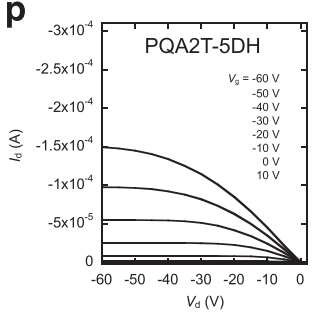

Figure 7 Transfer (a-d, $\mathbf{i}-\mathbf{l}$ ) and output (e-h, $\mathbf{m}-\mathbf{p}$ ) characteristics of typical OFET devices based on PTTD4Ts (a-h) and PQA2Ts (i-p). OFET, organic fieldeffect transistor. A full color version of this figure is available at Polymer Journal online.

Table 3 OFET characteristics of the polymers

\begin{tabular}{|c|c|c|c|c|}
\hline Polymer & $\mu_{h}\left(\mathrm{~cm}^{2} V^{-1} \mathrm{~s}^{-1}\right)^{\mathrm{a}}$ & $\mathrm{V}_{\text {th }}(V)$ & $\mu_{e}\left(c m^{2} V^{-1} s^{-1}\right)^{a}$ & $\mathrm{~V}_{t h}(V)$ \\
\hline PTTD4T-3DP & $0.11( \pm 0.02)$ & $-10.6( \pm 0.4)$ & $0.054( \pm 0.014)$ & $21.2( \pm 0.7)$ \\
\hline PTTD4T-4DH & $0.34( \pm 0.08)$ & $-6.3( \pm 1.6)$ & $0.16( \pm 0.04)$ & $21.0( \pm 1.2)$ \\
\hline PTTD4T-5DH & $0.20( \pm 0.03)$ & $-7.4( \pm 0.4)$ & $0.093( \pm 0.024)$ & $22.6( \pm 1.4)$ \\
\hline PQA2T-2DT & $0.16( \pm 0.02)$ & $-11.7( \pm 0.5)$ & NA & NA \\
\hline PQA2T-3DP & $0.15( \pm 0.02)$ & $-11.2( \pm 0.8)$ & NA & NA \\
\hline PQA2T-4DH & $0.33( \pm 0.04)$ & $-10.1( \pm 1.3)$ & NA & NA \\
\hline PQA2T-5DH & $0.22( \pm 0.04)$ & $-11.8( \pm 0.9)$ & NA & NA \\
\hline
\end{tabular}

Abbreviations: NA, not available; OFET, organic field-effect transistor.

${ }^{a}$ Average hole $\left(\mu_{h}\right)$ and electron $\left(\mu_{\mathrm{e}}\right)$ mobilities extracted from the saturation regime over more than 10 devices and their s.d.s. 
$-5 \mathrm{DH}$. This result indicates that the intermolecular interactions of the polymers increased, which was most likely due to reduced steric hindrance with the distance of the branching point regardless of the $\pi$-cores where the alkyl group was introduced. This change in the ordering structure is consistent with the change in the electronic properties described above.

We also performed atomic force microscopy on the polymer thin films (Figure 6). The atomic force microscopy images indicated that all of the thin films had smooth surfaces (root-mean-square roughness $<2 \mathrm{~nm}$ ) and similar step-and-terrace surface morphologies with step heights of approximately $2 \sim 3 \mathrm{~nm}$, which likely correspond to the lamellar spacing. This result also suggests that the polymers formed lamellar structures with edge-on orientations, and the morphology was independent of the branching position of the side chains.

To examine the charge transport properties of the polymers, bottom-gate-top-contact OFET devices using the polymer thin films were fabricated and characterized. The polymer thin films were deposited on octadecyltriethoxysilane-treated $\mathrm{Si} / \mathrm{SiO}_{2}$ substrates via spin-coating the polymer solutions followed by annealing at $300{ }^{\circ} \mathrm{C}$ in a glove box. Then, the source and drain gold electrodes, which have a channel length of $40 \mu \mathrm{m}$ and a channel width of $1500 \mu \mathrm{m}$, were vacuum deposited onto the polymer thin films. The typical transfer and output characteristics of the devices measured in air are shown in Figure 7. The PTTD4T-based devices exhibited V-shape transfer curves under both $\mathrm{p}$ - and n-channel operations, which are typical of ambipolar OFETs. However, the PQA2T-based devices worked only under p-channel operation. All the PTTD4T- and PQA2T-based devices exhibited good saturation behaviors in the output curves. The hole $\left(\mu_{\mathrm{h}}\right)$ and electron $\left(\mu_{\mathrm{e}}\right)$ mobilities of the OFET devices in the saturation regime $\left(V_{\mathrm{g}}=-60 \mathrm{~V}\right.$ for $\mathrm{p}$-channel operations or $+60 \mathrm{~V}$ for $n$-channel operation) and the threshold voltages were extracted from the transfer curves and are summarized in Table 3.

In PTTD4Ts, the average $\mu_{\mathrm{h}}$ and $\mu_{\mathrm{e}}$ ranged from 0.11 to $0.34 \mathrm{~cm}^{2} \mathrm{~V}^{-1} \mathrm{~s}^{-1}$ and 0.054 to $0.17 \mathrm{~cm}^{2} \mathrm{~V}^{-1} \mathrm{~s}^{-1}$, respectively, whereas in PQA2Ts, the $\mu_{\mathrm{h}}$ ranged from 0.15 to $0.33 \mathrm{~cm}^{2} \mathrm{~V}^{-1} \mathrm{~s}^{-1}$. In PTTD4Ts, a clear correlation was not observed between the mobility and the ordering structure. The polymers with a branching point farther from the backbone did not necessarily exhibit higher mobilities even though the $\pi-\pi$ stacking was enhanced. It is important to note that the device performance is sensitive to many other factors. ${ }^{34,35}$ Because the energy levels that determine the charge injection and morphology are similar regardless of the alkyl groups. We speculate that some other factors, such as interfacial structures, may affect the OFET performance more than the bulk ordering structure in PTTD4Ts. By contrast, the OFETs based on PQA2Ts exhibited a clear correlation between the $\mu_{\mathrm{h}}$ and the ordering structure. The $\mu_{\mathrm{h}}$ tended to increase as the $\pi-\pi$ stacking $d$-spacing decreased even though the mobility variation was moderate. Because the $\pi-\pi$ stacking $d$-spacing also exhibited a clear correlation with the branching position, the branching position is an important design parameter for developing high performance polymers.

\section{CONCLUSION}

We synthesized two series of semiconducting polymers (PTTD4Ts and PQA2Ts) bearing 2-decyltetradecyl (2DT), 3-decylpentadecyl (3DP), 4-decylhexadecyl $(\mathbf{4 D H})$, and 5-decylheptadecyl $(\mathbf{5 D H})$ groups as the side chain, in which the branching position was systematically moved away from the backbone. While the alkyl groups were introduced into the large $\pi$-core (QA unit) in PQA2Ts, they were introduced into the thiophene ring in PTTD4Ts. In the absorption spectra, although the absorption edge that corresponds to $E_{\mathrm{g}}$ did not change, the vibronic structure gradually changed (that is, the low-energy peak intensified) as the branching distance in both polymer systems increased. This result suggests an increased order in the polymer packing structure in the thin film. The GIXD measurement revealed that the $\pi-\pi$ stacking $d$-spacing gradually decreased as the branching position moved away from the backbone, indicating that the intermolecular interactions were enhanced due to suppression of the steric hindrance. Although a clear correlation between the branching position and the charge carrier mobility was not observed in PTTD4Ts, the charge carrier mobility increased as a function of the branching distance in PQA2Ts. These results indicate that the branching position affects the intermolecular interaction of the polymer backbones regardless of the substitution position or the $\pi$-cores where the alkyl groups were introduced. Therefore, careful choice of the branching position is required for semiconducting polymers to optimize the ordering structure and the charge transport property.

\section{CONFLICT OF INTEREST}

The authors declare no conflict of interest.

\section{ACKNOWLEDGEMENTS}

This work was supported by Grants-in-Aid for Scientific Research (24685030) from MEXT and the Strategic Promotion of Innovative Research and Development from JST. HRMS and EA were carried out at the Materials Characterization Support Unit in RIKEN, Advanced Technology Support Division. The DFT calculations were performed using the RIKEN Integrated Cluster of Clusters (RICC). The two-dimensional GIXD experiments were conducted at BL46XU of SPring-8 with the approval of the Japan Synchrotron Radiation Research Institute (Proposals 2014B1915 and 2015A1696). We thank Dr T. Koganezawa for support for the two-dimensional GIXD measurements. We also thank the TOSOH Corporation for the gel permeation chromatography measurements of PQA2Ts.

1 Sirringhaus, H., Brown, P. J., Friend, R. H., Nielsen, M. M., Bechgaard, K., Langeveld-Voss, B. M. W., Spiering, A. J. H., Janssen, R. A. J., Meijer, E. W., Herwig, P. \& de Leeuw, D. M. Two-dimensional charge transport in self-organized, high-mobility conjugated polymers. Nature 401, 685-688 (1999).

2 Burroughes, J. H., Bradley, D. D. C., Brown, A. R., Marks, R. N., Mackay, K., Friend, R. H., Burns, P. L. \& Holmes, A. B. Light-emitting diodes based on conjugated polymers. Nature 347, 539-541 (1990).

3 Yu, G., Gao, J., Hummelen, J. C., Wudl, F. \& Heeger, A. J. Polymer photovoltaic cells: enhanced efficiencies via a network of internal donor-acceptor heterojunctions. Science 270, 1789-1791 (1995).

4 Hou, J., Park, M.-H., Zhang, S., Yao, Y., Chen, L.-M., Li, J.-H. \& Yang, Y. Bandgap and molecular energy level control of conjugated polymer photovoltaic materials based on benzo[1,2-b:4,5-b']dithiophene. Macromolecules 41, 6012-6018 (2008).

$5 \mathrm{Li}, \mathrm{Y}$. Molecular design of photovoltaic materials for polymer solar cells: toward suitable electronic energy levels and broad absorption. Acc. Chem. Res. 45, 723-733 (2012).

6 Osaka, I. Semiconducting polymers based on electron-deficient [pi]-building units. Polym. J. 47, 18-25 (2015).

7 Bérubé, N., Gaudreau, J. \& Côté, M. Low band gap polymers design approach based on a mix of aromatic and quinoid structures. Macromolecules 46, 6873-6880 (2013).

8 Kitamura, C., Tanaka, S. \& Yamashita, Y. Design of narrow-bandgap polymers. Syntheses and properties of monomers and polymers containing aromatic-donor and o-Quinoid-acceptor units. Chem. Mater. 8, 570-578 (1996).

9 Koizumi, Y., Ide, M., Saeki, A., Vijayakumar, C., Balan, B., Kawamoto, M. \& Seki, S. Thienoisoindigo-based low-band gap polymers for organic electronic devices. Polym. Chem. 4, 484-494 (2013).

10 Chen, M. S., Niskala, J. R., Unruh, D. A., Chu, C. K., Lee, O. P. \& Fréchet, J. M. J. Control of Polymer-packing orientation in thin films through synthetic tailoring of backbone coplanarity. Chem. Mater. 25, 4088-4096 (2013).

11 McCullough, R. D. \& Lowe, R. D. Enhanced electrical conductivity in regioselectively synthesized poly(3-alkylthiophenes). J. Chem. Soc. Chem. Commun. 70-72 (1992).

12 McCullough, R. D., Lowe, R. D., Jayaraman, M. \& Anderson, D. L. Design, synthesis, and control of conducting polymer architectures: structurally homogeneous poly(3-alkylthiophenes). J. Org. Chem. 58, 904-912 (1993).

13 Chen, T. A. \& Rieke, R. D. The first regioregular head-to-tail poly(3-hexylthiophene-2,5diyl) and a regiorandom isopolymer: nickel versus palladium catalysis of 2(5)-bromo-5 
(2)-(bromozincio)-3-hexylthiophene polymerization. J. Am. Chem. Soc. 114, 10087-10088 (1992).

14 McCullough, R. D., Tristram-Nagle, S., Williams, S. P., Lowe, R. D. \& Jayaraman, M. Self-orienting head-to-tail poly(3-alkylthiophenes): new insights on structure-property relationships in conducting polymers. J. Am. Chem. Soc. 115, 4910-4911 (1993).

15 Osaka, I. \& McCullough, R. D. Advances in molecular design and synthesis of regioregular polythiophenes. Acc. Chem. Res. 41, 1202-1214 (2008).

$16 \mathrm{Mei}$, J. \& Bao, Z. Side chain engineering in solution-processable conjugated polymers. Chem. Mater. 26, 604-615 (2014)

17 Lei, T., Wang, J.-Y. \& Pei, J. Roles of flexible chains in organic semiconducting materials. Chem. Mater. 26, 594-603 (2014).

18 Osaka, I. \& Takimiya, K. Backbone orientation in semiconducting polymers. Polymer 59, A1-A15 (2015).

19 Osaka, I., Abe, T., Shimawaki, M., Koganezawa, T. \& Takimiya, K. Naphthodithiophenebased donor-acceptor polymers: versatile semiconductors for OFETs and OPVs. ACS Macro Lett. 1, 437-440 (2012).

20 Osaka, I., Zhang, R., Liu, J., Smilgies, D.-M., Kowalewski, T. \& McCullough, R. D. Highly stable semiconducting polymers based on thiazolothiazole. Chem. Mater. 22, 4191-4196 (2010).

21 Mei, J., Kim, D. H. Ayzner, A. L., Toney, M. F. \& Bao, Z. Siloxane-terminated solubilizing side chains: bringing conjugated polymer backbones closer and boosting hole mobilities in thin-film transistors. J. Am. Chem. Soc. 133, 20130-20133 (2011).

22 Kang, I., Yun, H.-J., Chung, D. S., Kwon, S.-K. \& Kim, Y.-H. Record high hole mobility in polymer semiconductors via side-chain engineering. J. Am. Chem. Soc. 135, 14896-14899 (2013)

23 Mei, J., Wu, H.-C., Diao, Y., Appleton, A., Wang, H., Zhou, Y., Lee, W.-Y., Kurosawa, T., Chen, W.-C. \& Bao, Z. Effect of spacer length of siloxane-terminated side chains on charge transport in isoindigo-based polymer semiconductor thin films. Adv. Funct. Mater. 25, 3455-3462 (2015).

24 Han, A. R., Dutta, G. K., Lee, J., Lee, H. R., Lee, S. M., Ahn, H., Shin, T. J., Oh, J. H. \& Yang, C. $\varepsilon$-Branched flexible side chain substituted diketopyrrolopyrrole-containing polymers designed for high hole and electron mobilities. Adv. Funct. Mater. 25, 247-254 (2015)
25 Fu, B., Baltazar, J., Sankar, A. R., Chu, P.-H., Zhang, S., Collard, D. M. \& Reichmanis, E. Enhancing field-effect mobility of conjugated polymers through rational design of branched side chains. Adv. Funct. Mater. 24, 3734-3744 (2014)

26 Lei, T., Dou, J.-H. \& Pei, J. Influence of alkyl chain branching positions on the hole mobilities of polymer thin-film transistors. Adv. Mater. 24, 6457-6461 (2012).

27 Dou, J.-H., Zheng, Y.-Q., Lei, T., Zhang, S.-D., Wang, Z., Zhang, W.-B., Wang, J.-Y. \& Pei, J. Systematic investigation of side-chain branching position effect on electron carrier mobility in conjugated polymers. Adv. Funct. Mater. 24, 6270-6278 (2014).

28 Back, J. Y., Yu, H., Song, I., Kang, I., Ahn, H., Shin, T. J., Kwon, S.-K., Oh, J. H. \& $\mathrm{Kim}$, Y.-H. Investigation of structure-property relationships in diketopyrrolopyrrolebased polymer semiconductors via side-chain engineering. Chem. Mater. 27, 1732-1739 (2015).

29 Mori, H., Suetsugu, M., Nishinaga, S., Chang, N.-H., Nonobe, H., Okuda, Y. \& Nishihara, Y. Synthesis, characterization, and solar cell and transistor applications of phenanthro[1,2-b:8,7-b']dithiophene-diketopyrrolopyrrole semiconducting polymers. J. Polym. Sci. A Polym. Chem. 53, 709-718 (2015).

30 Kawabata, K., Saito, M., Osaka, I. \& Takimiya, K. Very small bandgap $\pi$-conjugated polymers with extended thienoquinoids. J. Am. Chem. Soc. 138, 7725-7732 (2016).

31 Huynh, C., Derguini-Boumechal, F. \& Linstrumelle, G. Copper-catalysed reactions of grignard reagents with epoxides and oxetane. Tetrahedron Lett. 20, 1503-1506 (1979).

32 Kawabata, K., Osaka, I., Nakano, M., Takemura, N., Koganezawa, T. \& Takimiya, K. Thienothiophene-2,5-dione-based donor-acceptor polymers: improved synthesis and influence of the donor units on ambipolar charge transport properties. Adv. Electron. Mater. 1, 1500039 (2015).

33 Osaka, I., Akita, M., Koganezawa, T. \& Takimiya, K. Quinacridone-based semiconducting polymers: implication of electronic structure and orientational order for charge transport property. Chem. Mater. 24, 1235-1243 (2012).

34 Sirringhaus, H., Bird, M. \& Zhao, N. Charge transport physics of conjugated polymer field-effect transistors. Adv. Mater. 22, 3893-3898 (2010).

35 Sirringhaus, H. 25th Anniversary Article: organic field-effect transistors: the path beyond amorphous silicon. Adv. Mater. 26, 1319-1335 (2014).

Supplementary Information accompanies the paper on Polymer Journal website (http://www.nature.com/pj) 